\title{
Challenges in fibromyalgia diagnosis: from meaning of symptoms to fibromyalgia labeling
}

\author{
${ }^{1}$ Department of Rheumatology, Iran University of Medical Sciences, Tehran, \\ ${ }^{2}$ Rheumatology Research Center, Razi Hospital, School of Medicine, Guilan University of Medical Sciences, Rasht, \\ ${ }^{3}$ Otolaryngology and Head and Neck Surgery Research Center, Iran University of Medical Sciences, Tehran, Iran
}

Ali Bidari ${ }^{1}$ Banafsheh Ghavidel Parsa ${ }^{2}$, and Babak Ghalehbaghi ${ }^{3}$

\begin{abstract}
Fibromyalgia (FM) is a contested illness with ill-defined boundaries. There is no clearly defined cut-point that separates FM from non-FM. Diagnosis of FM has been faced with several challenges that occur, including patients' health care-seeking behavior, symptoms recognition, and FM labeling by physicians. This review focuses on important but less visible factors that have a profound influence on under- or over-diagnosis of FM. FM shows different phenotypes and disease expression in patients and even in one patient over time. Psychosocial and cultural factors seem to be a contemporary ferment in FM which play a major role in physician diagnosis even more than having severe symptom levels in FM patients. Although the FM criteria are the only current methods which can be used for classification of FM patients in surveys, research, and clinical settings, there are several key pieces missing in the fibromyalgia diagnostic puzzle, such as invalidation, psychosocial factors, and heterogeneous disease expression. Regarding the complex nature of FM, as well as the arbitrary and illusory constructs of the existing FM criteria, FM diagnosis frequently fails to provide a clinical diagnosis fit to reality. A physicians' judgment, obtained in real communicative environments with patients, beyond the existing constructional scores, seems the only reliable way for more valid diagnoses. It plays a pivotal role in the meaning and conceptualization of symptoms and psychosocial factors, making diagnoses and labeling of FM. It is better to see FM as a whole, not as a medical specialty or constructional scores. (Korean J Pain 2018; 31: 147-54)
\end{abstract}

Key Words: Chronic fatigue syndrome; Chronic pain; Classification; Diagnosis; Dyssomnia; Fibromyalgia; Headache; Musculoskeletal diseases; Patient selection; Reproducibility of results; Surveys and questionnaires.

\section{INTRODUCTION}

Fibromyalgia (FM) is a contested illness where there is a considerable controversy in its nature, existence, assessment, and diagnosis [1-3]. There are several challenges in
FM diagnosis that occur across patients' behavior in medical care seeking, to the meaning and conceptualization of symptoms by clinicians, and finally in making a diagnosis and the labeling of FM.

This review has focused on important but less visible

Received November 29, 2017. Revised February 8, 2018. Accepted February 19, 2018.

Correspondence to: Banafsheh Ghavidel Parsa

Rheumatology Research Center, Razi Hospital, School of Medicine, Guilan University of Medical Sciences, Sardar Jangal St., Rasht, Guilan 41448-95655, Iran

Tel: +98-9123277268, Fax: +98-1333530169, E-mail: bghavidelparsa@gmail.com

(a) This is an open-access article distributed under the terms of the Creative Commons Attribution Non-Commercial License (http:// creativecommons.org/licenses/by-nc/4.0/), which permits unrestricted non-commercial use, distribution, and reproduction in any medium, provided the original work is properly cited.

Copyright (c) The Korean Pain Society, 2018 
factors that influence profoundly on under- or over-diagnosis of FM by physicians. We hope this review creates a new gate to a holistic and real understanding of FM diagnosis beyond existing arbitrary and constructional scores.

\section{MAIN BODY}

\section{Fuzzy boundaries of fibromyalgia with broad and time-changing phenotypes}

Chronic widespread pain, sleep problems or unrefreshing sleep, physical exhaustion, and cognitive difficulties are the core symptoms of FM $[4,5]$. However, most patients diagnosed with FM report a wide range of additional somatic and psychological symptoms [4]. It has fuzzy boundaries that are cross-linked to other illness (such as psychological disorders) as subsets, mistaken diagnosis and comorbid conditions [2]. There are more confusing issues when we review our knowledge about FM neurobiology, illness definition, and accepted diagnostic criteria.

Although not yet fully understood, the new concept suggests that FM is a heterogeneous condition which likely has multiple potential etiologies [6]. It is important to not consider FM as 'yes' or 'no' but rather as the end of a continuum [6-8]. On the one end of this continuum is a purely peripherally driven painful condition and the other end of the continuum is when pain is nearly completely the result of altered central augmentation [6].

In fact, many clinical researches suggest that various individuals with chronic pain, including FM, are at various points in this continuum $[9,10]$. Thus, some patients may have stronger peripheral than central components, some mixed components, and many have stronger central components. Consequently, FM Patients may present with different phenotypes according to their different underlying neurobiology [6].

Furthermore, it is also debated whether patients maintain their characteristic phenotype and disease expression with time, or whether disease expression changes [11]. It is not surprising that an individual FM core symptom may emerge or decline as a predominant symptom over time [12].

Due to diversity of symptoms presentation and severity over time, it is possible that an individual with FM seeks medical help and is diagnosed with the FM label at one time, and the same patient, at another time, is given a diagnostic label that merely connotes more local complaints, such as chronic low back pain, headache, or temporomandibular joint disorder, irritable bowel syndrome (IBS), and so on $[6,13]$. It seems that some FM core symptoms diminish or fade over time [14], or patients do not mention actively in their core symptoms which may be non-significant or may be missed by the clinician.

Core symptom variation both within a patient over time as well as between patients in the setting of etiologic and symptom heterogeneity produces considerable perplexity in FM diagnosis, even in hands of experts. The clinicians who are familiar with FM contend that "they can recognize FM", but experts still debate practical clinical diagnostic criteria $[11,15]$. So, the precise diagnosis in an individual patient may be elusive, with symptoms present for years leading to many health care encounters and diagnostic delay [16].

\section{Patients and physicians' confounding factors in FM diagnosis}

The substantial role for psychosocial and environmental determinants in FM patients capturing and diagnosis cannot be denied [17]. It was demonstrated that having the severe core symptoms that define FM is not essential to receiving an FM diagnosis. Rather, demographic and social disadvantage appears to be more important than symptom severity in making FM clinical diagnosis by clinicians [17]. For obtaining an FM diagnosis, symptomatic persons must seek medical care from clinicians and those clinicians must interpret and label the described symptoms as being FM. A patient cannot obtain an FM diagnosis unless they want to see a clinician who is willing to make that diagnosis. So, clinical diagnosis of FM patients is necessarily confounded by patient's health care seeking behavior and clinical selection [18].

Furthermore, in a clinical setting, physicians' beliefs and biases influence FM diagnosis [2]. All FM assessments are subjective and there is no clear gold standard for FM diagnosis [19]. The constellation of severe symptoms can be clinically interpreted and diagnosed in many different ways, perhaps influenced by clinicians [20]. This leads to a large number of apparently over- and under-diagnosed subjects in clinical settings $[2,17]$. On one hand, a diagnosis of FM can legitimize vague and difficult symptoms, allowing entrée into official diagnosis or providing a way 
toward official disability status. So, resultantly, millions of people may be given a FM diagnosis without satisfying the severity cutoff of FM diagnosis [2,21].

On the other hand, it appears that many clinicians, when faced with the opportunity to diagnose FM may miss making such a diagnosis. This action may be related to the complexity and diversity of FM symptoms, the presence of other medical diagnoses, a lack of knowledge; or a disagreement about the nature and meaning of symptoms and how they should be interpreted [18,22]. So, it is not so surprising to see patients who have gone from doctor to doctor and who underwent multiple diagnostic tests and received alternative diagnoses such as lupus erythematous, rheumatoid arthritis, non-specific "arthritis" and malingering $[16,23]$.

FM patients may experience and mention some level of lack of understanding or discounting by their clinicians, family, or other people that interact with them. The term "invalidation" is used to describe the patients' perception that their illness and symptoms is not recognized by their social environment [24]. Although invalidation is not exclusive to FM patients and also affects other rheumatic diseases, it most often perceived and expressed by $\mathrm{FM} \mathrm{pa}^{-}$ tients $[25,26]$. Practicing clinicians are often confronted with a high prevalence of invalidation in FM patients. In recent years, some researchers have suggested that $\mathrm{pa}^{-}$ tients' perception of invalidation by clinicians could be a clue for the diagnosis of FM. In fact, it would certainly be helpful to be clear whether invalidation assessment may be useful for diagnosis or classification of FM [27].

Resultantly, it seems that both patients' behavior and clinical symptoms as well as physicians' symptom interpretation, beliefs, invalidation, or intimacy with patients can clearly influence whether or not an FM diagnosis is given.

\section{Application of the FM classification criteria in our diagnostic lexicon}

The existing FM classification criteria has clearly altered and introduced a more precise case definition of FM in the recent decades. The recent criteria for the diagnosis of FM are clearly a steps towards a better understanding of this condition and are currently used for survey and clinical purposes. However, it seems much more effort must be made in this direction. The existing diagnostic criteria don't seem to cover the broad heterogeneity and variation in symptoms and severity.

Regarding the intuitive complexity of the nature of FM, as well as the presence of these broad confounding factors in diagnosis, using a more valid diagnostic instrument seems essential. It seems that FM is better conceptualized as a symptom and etiological continuum rather than as a discreet dichotomous entity inferred by existing criteria [8,28-31].

No clear distinguishing point exists where FM stops being FM and becomes some other illness with more localized complaints, or no illness at all [18]. This raises a fundamental question about how diagnostic labeling should be applied according to the diagnostic criteria, because one patient may be labeled as FM on one occasion and non-FM on another. FM patients often move on this spectrum, occasionally toward a typical FM phenotype and at other times toward a normal phenotype [12], probably due to the effects of certain psychological, environmental, or other unknown factors. But, it is important to note that each patient is the same person in regards to genotype, neurobiology, and other risk factors.

Although, as mentioned before, psychological and environmental, or socio-cultural factors also play an important role in presentation, recognition and capturing, or even of labeling FM cases $[17,18]$, there is no place for these issues in the FM criteria. In other words, these factors undoubtedly influence diagnosis of FM and are even more important than symptom severity in the probability of receiving an FM diagnosis by medical healthcare providers [17].

On the other hand, physicians will differ in their interpretation of patient complaints and also the severity of symptoms. A physician both records the complaint as presented by the patient, and also assesses the validity of the symptom report which the latter does not provide by the existing criteria. It is likely that some persons with FM may exaggerate subjective symptoms for reasons of secondary gain such as obtaining disability payments [32].

So, full clinical evaluation such as assessment of dayto-day activities, information from all treating healthcare professionals, and careful observation during assessment for evidence of discrepancies in clinical findings should still remain the standard of patient evaluation and diagnosis, rather than relying on differently interpreted or perceived FM criteria items. 
Regarding both the above-mentioned issues and some other points in criticizing the existing criteria (see "Arbitrary and illusory constructs of the existing FM criteria"), it seems these criteria have not met expectations. There is a critical question which deals with the existing criteria: to what extent are the symptom-based and arbitrary constructs of the existing FM criteria suitable as diagnostic instruments? Although these criteria have incorporated most core symptoms of FM, there appears to remain a long and hard path to achieve a holistic and intuitive instrument for FM diagnosis.

In Egolff view, "No specialist in internal medicine would be likely to confuse the New York Heart Association (NYHA) criteria for quantifying dyspnea in heart failure with the diagnosis of the underlying cardiomyopathy" [13]. In other words, use of symptom scores and cutoff points are not the type of instrument needed or suitable for the clinical diagnosis of diseases. It seems that the just symptom-based approach for diagnosis of disease was subject to criticism from the very beginning $[13,33]$. This approach results in a failure to provide a diagnostic instrument fit for clinical purposes [7].

Diagnosis of FM is discretionary and patient symptoms level, psychosocial factors, and external societal factors influence that discretion [2]. It is wise to quote George Ehrlich's sentence: "When one has tuberculosis, one has tuberculosis, whether or not it is diagnosed, but "no one has FM until it is diagnosed" [34]. So, diagnostic labeling in FM must be used with caution and discretion [17,34]. This double-edged tool can have both beneficial and harmful effects. On the one hand, delay in labeling can result in excessive testing, inappropriate treatment, and consequently economic burden on the healthcare system and frustration for patients and their families. On the other hand, extensive use of FM diagnosis as an explanation of mild-to-moderate levels of symptoms or illness impact has likely led to substantial harm to patient and societal costs [17].

Finally, at the present time, no matter where we put FM criteria in our diagnostic lexicon, undoubtedly, these criteria are the only current methods which can be used for classification of FM patients in surveys, research, and clinical settings. However, there are several missing pieces in the diagnostic puzzle of FM. Until receiving more accurate data about diagnostic methods, it seems that there is no way except the discretionary policy of clinicians for the diagnosis and labeling of this disorder. Physicians' judgment obtained in a real communicative environment with patients, beyond the existing constructional scores, seems the only reliable way for more valid diagnoses. Much more effort is required to identify the many missing pieces of the FM puzzle, which seem to cause and perpetuate this medical problem.

\section{Substantial role of psychosocial factors in FM diagnosis}

Data obtained from large population studies has provided new insight into FM diagnosis in the general population and clinical settings $[17,18]$. It revealed a fundamental role for psychosocial and cultural factors in the diagnostic acceptability of FM. These studies showed that having severe symptoms which define FM showed substantially less influence on how patients received FM diagnosis by physicians. Rather, health care-seeking behavior and clinical selection, as well as social and cultural factors are more important in physician diagnosis. So, $73 \%$ of the population who had enough severe symptoms to meet a criteria-based diagnosis did not receive a clinical diagnosis of FM [17]. On the other hand, $75 \%$ of persons in the US population reporting a clinician diagnosis of fibromyalgia did not satisfy FM criteria [18]. This observation showed the large numbers of apparently over- and under-diagnosed subjects. In this regard, demographic factors and social disadvantage appears to be more important than symptom reporting in predicting FM diagnosis [17]. It seems the under- and over-diagnosis of clinical FM may be at least partly related to the possible influence of social and cultural factors.

To have clinical diagnosis and to legitimize the illness, which does not have any visible sign of disease, a patient must receive an insurance diagnosis by physician [35]. In developed countries, FM patients often take an active role in their diagnosis, recognizing their poly-symptomatic distress (PDS) as FM and seeking clinical care for confirmation and treatment [21].

As a result, the diagnosis of FM has been extended beyond expectations and consequently has lead to an over-diagnosis of this illness [18]. Self-reported clinical FM diagnosis appears to be increased by programs for clinical awareness, through educational activities for clinicians, academic research, patient advocacy, and directto-patient advertising, much of which has been financed 
by the pharmaceutical industry $[1,21,36]$.

In contrast, the substantial numbers of under-diagnosed FM cases showed high level of invalidation of $\mathrm{pa}^{-}$ tients by their medical care providers and social environment. Furthermore, many physicians express frustration directed not only at the FM issue, but also at patients. They will not see patients that are referred to them for suspicious FM and others will only see the patient for a one-time assessment to exclude other disorders [34]. This misunderstanding can also be seen in family members or other people interacting with patients [24,27]. It seems that this high level of invalidation may be related to the great psychological distress expressed by FM patients and also to inherently invisible FM symptoms and to sociocultural factors [25].

Furthermore, the surprising data about substantial discordance between physician-based and criteria-based diagnosis raise a simple but essential question concerning to what extent we are permitted to confine all of our discretion about symptoms' meaning and severity into the scores or criteria construction. Undoubtedly, the answer to this question can be an important step toward a more real diagnosis of FM.

\section{Arbitrary and illusory constructs of the existing FM criteria}

In 1990, The ACR committee found that the presence of widespread pain combined with at least 11 out of 18 tender points best separated patients with fibromyalgia from controls that led to development of the 1990 ACR criteria [28]. However, tender point examination was hard for most non-rheumatologists to perform [20,29]. Numerous concerns were raised on the reliability and validity of the tender points in the clinical setting, which eventually led to stopping their use in clinics [20].

So, the 2010 ACR preliminary diagnostic criteria proposed to address numerous problems with the 1990 ACR criteria [29]. The ACR 2010 criteria not only eliminated tender points, they also changed the case definition of FM to an illness characterized by self-reported, multiple painful regions (0-19 WPI) and additional key symptoms, such as problems with fatigue, sleep, cognition, and the extent of somatic symptom reporting [29].

The change in definition from 1990 to 2010 gave an important role to non-pain symptoms in FM diagnosis.
Furthermore, there were a small proportion of patients whom clinicians diagnosed as having FM but who did not satisfy the 1990 criteria because of having either $<11$ tender points or slightly less than the full ACR 1990 definition of widespread pain [29]. These cases were recognized simply by the 2010 ACR criteria. Moreover, another remarkable achievement of the 2010 criteria was introducing of the Poly-symptomatic distress (PSD) scale [7]. With the PSD Scale, dichotomizing diagnosis and distress becomes less important [7,20].

Because it shows where the patient is on the continuum of distress or symptoms, as well as how far the patient is from the positive/negative dividing line [30]. Although the 2010 ACR criteria mapped out FM as a dimensional or continuum disorder, this concept did not apply completely. There is still a cut point for FM diagnosis in the PSD or the 2010 ACR criteria where patients are classified as FM versus non-FM. Hence this fact raises a fundamental question about how much difference there truly is between patients on different side of the cut point. In fact, the boundaries of FM are not well defined and there is no clearly defined cut point that separates FM from non-FM $[2,30]$. Moreover, all assessments are subjective, and both physicians and patients might differ in their assessment of severity [20]. So, this makes distinguishing cases and non-cases difficult and arbitrary [1].

Additionally, it seems that dropping the tender point concept is not only the main advantage of the ACR 2010 criteria but also their greatest drawback [13]. The 2010 criteria study showed that muscle tenderness was one of the most important variables classifying cases and non-cases of FM, although tenderness was not used in the final formulation of the criteria [29]. Numerous previous studies confirm that the feature of hyperalgesia is a crucial and intuitive characteristic of FM [1,3,37]. Although the majority of experts agree that tender points' examination and interpretation is difficult and may miss the intended target $[20,29]$, there are still some valid concerns that totally abandoning the tender point concept would omit examination of the distinctive 'hyperalgesia aspect' of FM [13].

Additionally, the 2010 criteria are inherently underrepresentative in regards to the nature of the pain. It only incorporates pain regions or distribution and does not consider pain quality and even quantity [3]. Evaluation of pain is multidimensional and fewer pain sites (fewer WPI) will 
not necessarily connote to lower pain significance or impact [38]. So, reliance just on the pain site numbers for assessment of pain impact is not the right way to a valid diagnosis.

On the other hand, non-pain symptoms were selected from a larger set of symptoms, based on empirical-statistical criteria according to their importance in distinguishing patients with FM from those who are non-FM [29]. This larger set did not include symptoms that had been found to be essential to FM recognition, for example, stiffness, balance, tenderness to touch, environmental sensitivity, and invalidation $[3,29,31]$.

Ultimately, no criteria can translate a real understanding of patient's symptoms. Comprehension of this fact is more important and perplexing since all the diagnostic methods of assessment are subjective.

The misclassification rate of the criteria was significant among different populations [39-44], especially when it was applied to the patients with regional pain disorders in tertiary pain clinics [13]. These observations lead to the revision of the 2010 ACR criteria and to developing the 2016 modified criteria. The researchers attempted to impose the requirement of a meeting of widespread pain criterion, such as the 1990 criterion but with caution to take away the restrictions of the 1990 criterion [30]. It seems that it is time to criticize changing the criteria. Has changing or revising the criteria over the past decades satisfied researchers or clinicians for making a valid diagnosis of FM? The answer is probably "no". Contrary to the declared claim of these criteria, they are not simple and practical for clinical use.

Most practitioners don't use any criteria for FM diagnosis in the clinical settings $[2,17]$. This may be related to the arbitrary nature of these criteria or the criteria's limitations in coverage of all FM characteristics. It seems they are not representative of the multidimensionality and complexity of the nature of FM. So, although the existing criteria can be useful in the recognition of FM, especially for research purposes, they are not able to replace the discretionary and holistic eye of expert practitioners.

\section{Labeling of FM diagnosis}

FM is a real but untenable diagnosis [34]. Diversity of symptoms and phenotypes even in one patient may lead to numerous labels over time by medical care providers
[6,16]. Furthermore, medicalization and a diagnostic expansion in a population with mild symptoms raise serious concerns about FM labeling. In developed countries, FM patients often take an active role in their diagnosis [17]. It is not uncommon to see self-diagnosis of FM [21]. The agency of social and electronic media and support groups extend this concept $[35,36]$. The existing data make clear that both under- and over-labeling can lead to personal and societal burdens with respect to economic and clinical aspects [16]. So, to return to the statement that "no one has FM until it is diagnosed and labeled", it is clear that a cautious and holistic approach must be emphasized in regard to patients with suspicious FM.

Modern medicine has empowered societies, physicians, and patients with vast increases in access to medical information, investigations, and with the ability to have more options about diagnoses and treatments [45]. However, it might be thought that little has changed after many years of research on FM diagnosis or treatment. Here, there is a quote to Frederick Wolfe: "A kind, conscientious physician treating a FM patient in 1980 or 1990 will have done as well as the 2016 health workers with access to all of the new publications and expensive if not very efficacious medications" [46].

\section{CONCLUSIONS}

Precise fibromyalgia diagnosis may be elusive. It seems that the meaning of FM symptoms and the conceptualization of patients' complaints in a logical manner needs to challenge all of experience and discretion of practicing physicians. This means that such logical and complex diagnostic challenges cannot be placed into the limited capacity of FM criteria.

Although these criteria are the only current methods which can be used for classification of FM patients in surveys, research, and clinical settings, there are several key pieces missing in the fibromyalgia diagnostic puzzle, such as invalidation, psychosocial factors, and heterogeneous disease expression.

Deciding if the patient labeled as having FM or not, and also evaluation of the FM patient for disease impact require a meticulous and discretionary approach to FM. It is better to see FM as a whole, and not as a medical specialty or constructional scores. 


\section{ACKNOWLEDGEMENTS}

This work was supported by the Deputy for Research of Guilan University of Medical Sciences, Rasht, Iran.

\section{REFERENCES}

1. Wolfe F, Walitt B. Culture, science and the changing nature of fibromyalgia. Nat Rev Rheumatol 2013; 9: 751-5.

2. Wolfe F, Walitt B. Fibromyalgia: a short commentary. J Headache Pain Manag 2016; 1: 27.

3. Schmidt-Wilcke T, Clauw DJ. Fibromyalgia: from pathophysiology to therapy. Nat Rev Rheumatol 2011; 7: 518-27.

4. Mease PJ, Arnold LM, Crofford LJ, Williams DA, Russell IJ, Humphrey $L$, et al. Identifying the clinical domains of fibromyalgia: contributions from clinician and patient Delphi exercises. Arthritis Rheum 2008; 59: 952-60.

5. Vincent A, Hoskin TL, Whipple MO, Clauw DJ, Barton DL, Benzo RP, et al. OMERACT-based fibromyalgia symptom subgroups: an exploratory cluster analysis. Arthritis Res Ther 2014; 16: 463.

6. Sluka KA, Clauw DJ. Neurobiology of fibromyalgia and chronic widespread pain. Neuroscience 2016; 338 : 114-29.

7. Wolfe F, Clauw DJ, Fitzcharles MA, Goldenberg DL, Häuser W, Katz RS, et al. Fibromyalgia criteria and severity scales for clinical and epidemiological studies: a modification of the ACR preliminary diagnostic criteria for fibromyalgia. J Rheumatol 2011; 38: 1113-22.

8. Wolfe F, Brähler E, Hinz A, Häuser W. Fibromyalgia prevalence, somatic symptom reporting, and the dimensionality of polysymptomatic distress: results from a survey of the general population. Arthritis Care Res (Hoboken) 2013; 65: 777-85.

9. Kosek E, Ordeberg G. Lack of pressure pain modulation by heterotopic noxious conditioning stimulation in patients with painful osteoarthritis before, but not following, surgical pain relief. Pain 2000; 88: 69-78.

10. Clauw DJ. Fibromyalgia: a clinical review. JAMA 2014; 311 : 1547-55.

11. Häuser W, Clauw DJ, Fitzcharles MA. Treatto-target strategy for fibromyalgia: opening the dialogue. Arthritis Care Res (Hoboken) 2017; 69: 462-6.

12. Bidari A, Ghavidel-Parsa B, Ghalehbaghi B. Reliability of ACR criteria over time to differentiate classic fibromyalgia from nonspecific widespread pain syndrome: a 6-month prospective cohort study. Mod Rheumatol 2009; 19: 663-9.

13. Egloff N, von Känel R, Müller V, Egle UT, Kokinogenis G, Lederbogen $\mathrm{S}$, et al. Implications of proposed fibromyalgia criteria across other functional pain syndromes. Scand J Rheumatol 2015; 44: 416-24.
14. Walitt B, Fitzcharles MA, Hassett AL, Katz RS, Häuser W, Wolfe F. The longitudinal outcome of fibromyalgia: a study of 1555 patients. J Rheumatol 2011; 38: 2238-46.

15. Perrot S, Choy E, Petersel D, Ginovker A, Kramer E. Survey of physician experiences and perceptions about the diagnosis and treatment of fibromyalgia. BMC Health Serv Res 2012; 12: 356.

16. Ghavidel-Parsa B, Bidari A, Amir Maafi A, Ghalebaghi B. The iceberg nature of fibromyalgia burden: the clinical and economic aspects. Korean J Pain 2015; 28: 169-76.

17. Walitt B, Katz RS, Bergman MJ, Wolfe F. Three-quarters of persons in the US population reporting a clinical diagnosis of fibromyalgia do not satisfy fibromyalgia criteria: the 2012 National Health Interview Survey. PLoS One 2016; 11: e0157235

18. Walitt B, Nahin RL, Katz RS, Bergman MJ, Wolfe F. The prevalence and characteristics of fibromyalgia in the 2012 National Health Interview Survey. PLoS One 2015; 10: e0138024.

19. Häuser W, Ablin J, Fitzcharles MA, Littlejohn G, Luciano JV, Usui C, et al. Fibromyalgia. Nat Rev Dis Primers 2015; 1 : 15022.

20. Wolfe F. Editorial: the status of fibromyalgia criteria. Arthritis Rheumatol 2015; 67: 330-3.

21. Wolfe F, Fitzcharles MA, Goldenberg DL, Häuser W, Katz RL, Mease PJ, et al. Comparison of physician-based and patient-based criteria for the diagnosis of fibromyalgia. Arthritis Care Res (Hoboken) 2016; 68: 652-9.

22. Aronowitz RA. When do symptoms become a disease? Ann Intern Med 2001; 134: 803-8.

23. Marchesoni A, De Marco G. Merashli M, McKenna F. Tinazzi I, Marzo-Ortega $\mathrm{H}$, et al. The problem in differentiation between psoriatic-related polyenthesitis and fibromyalgia. Rheumatology (Oxford) 2018; 57: 32-40.

24. Kool MB, van Middendorp $H$, Boeije HR, Geenen R. Understanding the lack of understanding: invalidation from the perspective of the patient with fibromyalgia. Arthritis Rheum 2009; 61: 1650-6.

25. Santiago MG, Marques A, Kool M, Geenen R, da Silva JA. Invalidation in patients with rheumatic diseases: clinical and psychological framework. J Rheumatol 2017; 44: 512-8.

26. Kool MB, van Middendorp H, Lumley MA, Schenk Y, Jacobs JW, Bijlsma JW, et al. Lack of understanding in fibromyalgia and rheumatoid arthritis: the IIness Invalidation Inventory (3*). Ann Rheum Dis 2010; 69: 1990-5.

27. Ghavidel-Parsa B, Amir Maafi A, Aarabi Y, Haghdoost A, Khojamli M, Montazeri A, et al. Correlation of invalidation with symptom severity and health status in fibromyalgia. Rheumatology (Oxford) 2015; 54: 482-6.

28. Wolfe F, Smythe HA, Yunus MB, Bennett RM, Bombardier C, Goldenberg DL, et al. The American College of Rheumatology 1990 criteria for the classification of fibromyalgia. 
Report of the Multicenter Criteria Committee. Arthritis Rheum 1990; 33: 160-72.

29. Wolfe F, Clauw DJ, Fitzcharles MA, Goldenberg DL, Katz RS, Mease P, et al. The American College of Rheumatology preliminary diagnostic criteria for fibromyalgia and measurement of symptom severity. Arthritis Care Res (Hoboken) 2010; 62: 600-10

30. Wolfe F, Clauw DJ, Fitzcharles MA, Goldenberg DL, Häuser W, Katz RL, et al. 2016 revisions to the 2010/2011 fibromyalgia diagnostic criteria. Semin Arthritis Rheum 2016; 46: 319-29.

31. Bennett RM, Friend R, Marcus D, Bernstein C, Han BK, Yachoui $\mathrm{R}$, et al. Criteria for the diagnosis of fibromyalgia: validation of the modified 2010 preliminary American College of Rheumatology criteria and the development of alternative criteria. Arthritis Care Res (Hoboken) 2014; 66: 1364-73.

32. Fitzcharles MA, Ste-Marie PA, Rampakakis E, Sampalis JS, Shir $Y$. Disability in fibromyalgia associates with symptom severity and occupation characteristics. J Rheumatol 2016; 43: 931-6.

33. Smythe HA. Unhelpful criteria sets for "diagnosis" and "assessment of severity" of fibromyalgia. J Rheumatol 2011; 38: 975-8.

34. Ehrlich GE. Pain is real; fibromyalgia isn't. J Rheumatol 2003; 30: 1666-7.

35. Hadler NM, Greenhalgh S. Labeling woefulness: the social construction of fibromyalgia. Spine (Phila Pa 1976) 2005: 30: $1-4$.

36. Barker KK. Listening to Lyrica: contested illnesses and pharmaceutical determinism. Soc Sci Med 2011; 73: 833-42.

37. Desmeules JA, Cedraschi C, Rapiti E, Baumgartner E, Finckh A, Cohen P, et al. Neurophysiologic evidence for a central sensitization in patients with fibromyalgia. Arthritis Rheum 2003; 48: 1420-9.

38. Friend R, Bennett RM. A critical examination of the Polysymptomatic Distress Scale construct as a symptom severity questionnaire. J Rheumatol 2015; 42: 1364-7.

39. Usui C, Hatta K, Aratani S, Yagishita N, Nishioka K, Kanazawa $T$, et al. The Japanese version of the modified ACR preliminary diagnostic criteria for fibromyalgia and the fibromyalgia symptom scale: reliability and validity. Mod Rheumatol 2013; 23: 846-50.

40. Jones GT, Atzeni F, Beasley M, Flüß E, Sarzi-Puttini P, Macfarlane GJ. The prevalence of fibromyalgia in the general population: a comparison of the American College of Rheumatology 1990, 2010, and modified 2010 classification criteria. Arthritis Rheumatol 2015; 67: 568-75.

41. Ferrari R, Russell AS. A questionnaire using the modified 2010 American College of Rheumatology criteria for fibromyalgia: specificity and sensitivity in clinical practice. J Rheumatol 2013; 40: 1590-5.

42. Bidari A, Hassanzadeh M, Ghavidel Parsa B, Kianmehr N, Kabir A, Pirhadi S, et al. Validation of the 2010 American College of Rheumatology preliminary diagnostic criteria for fibromyalgia in an Iranian population. Rheumatol Int 2013; 33: 2999-3007.

43. Häuser W, Jung E, Erbslöh-Möller B, Gesmann M, KühnBecker $H$, Petermann F, et al. Validation of the Fibromyalgia Survey Questionnaire within a cross-sectional survey. PLoS One 2012; 7: e37504.

44. Segura-Jiménez V, Aparicio VA, Álvarez-Gallardo IC, Soriano-Maldonado A, Estévez-López F, Delgado-Fernández M, et al. Validation of the modified 2010 American College of Rheumatology diagnostic criteria for fibromyalgia in a Spanish population. Rheumatology (Oxford) 2014; 53: 1803-11.

45. Furedi $F$. The end of professional dominance. Society 2006 ; 43: $14-8$.

46. Wolfe F. Most fibromyalgia research is worthless [Internet]. [place unknown]: The Fibromyalgia Perplex; 2016 [cited 2017 Oct 23]. Available at http://www.fmperplex.com/ 2016/08/30/most-fibromyalgia-research-is-worthless/. 